\title{
RENDIMENTO ACADÊMICO E ESTILOS DE APRENDIZAGEM: UM ESTUDO NA DISCIPLINA ANÁLISE DE CUSTOS
}

\author{
ACADEMIC PERFORMANCE AND LEARNING STYLES: A STUDY IN THE DISCIPLINE OF COST ANALYSIS \\ RENDIMIENTO ACADÉMICO Y ESTILOS DE APRENDIZAJE: UN ESTUDIO EN LA MATERIA DE ANÁLISIS \\ DE COSTOS
}

\author{
LARA FABIANA MORAIS BORGES \\ Mestre \\ Universidade Federal de Uberlândia - Brasil \\ larafaborges@gmail.com \\ ORCID: https://orcid.org/0000-0001-6320-0083 \\ EDVALDA ARAÚJO LEAL \\ Doutora \\ Universidade Federal de Uberlândia - Brasil \\ edvalda@ufu.br \\ ORCID: https://orcid.org/0000-0002-7497-5949 \\ TAÍS DUARTE SILVA \\ Doutoranda \\ Universidade Federal de Uberlândia - Brasil \\ taisduartes@yahoo.com.br \\ ORCID: https://orcid.org/0000-0002-5972-8851 \\ JANSER MOURA PEREIRA \\ Doutor \\ Universidade Federal de Uberlândia - Brasil \\ janser@ufu.br \\ ORCID: https://orcid.org/0000-0002-4622-6203
}

Submetido em: 28/12/2017

Aprovado em: 11/09/2018

Doi: alcance.v25n2(Mai/Ago).p161-176

\begin{abstract}
RESUMO
O objetivo do estudo foi verificar se o estilo de aprendizagem impacta no rendimento acadêmico dos alunos que cursaram a disciplina Análise de Custos no curso de Ciências Contábeis no que tange às avaliações formativas e somativas. Oportunamente, compararam-se a associação do rendimento na referida disciplina e o rendimento do aluno no semestre, bem como com o coeficiente geral do curso. Para a análise do rendimento acadêmico, utilizouse a Teoria da Avaliação. Para a coleta de dados, o instrumento utilizado, baseado na literatura, possibilitou mensurar o estilo de aprendizagem. Para a análise dos dados, utilizaram-se a estatística descritiva e o teste de Wilcoxon. A amostra compreende 111 alunos matriculados no segundo semestre do ano de 2015 e primeiro semestre de 2016, representando $88 \%$ da população. Os resultados apontam a predominância dos estilos ativo, sensorial, visual e sequencial. Quanto ao rendimento acadêmico, verificaram-se maiores médias para as avaliações individuais (somativas). Na comparação do rendimento e os estilos de aprendizagem dos alunos, identificou-se um equilibrio nas dimensões, ou seja, as médias são próximas tanto para avaliações individuais
\end{abstract}


quanto em grupo e, também, nas médias do Coeficiente de Rendimento Acadêmico (CRA) semestral e geral. 0 teste não paramétrico Wilcoxon indicou não haver diferença na maioria dos resultados, com exceção entre a avaliação somativa e o estilo sequencial/global.

Palavras chave: Estilos de Aprendizagem. Rendimento Acadêmico. Ciências Contábeis.

\section{ABSTRACT}

The aim of this study was to investigate whether learning style impacts academic performance in the formative and summative evaluations of students of the Cost Analysis in Accounting Sciences course. We compared the association of performance in this discipline and the student's grades for the semester, as well as with the general coefficient of the course. For the analysis of the academic results, Evaluation Theory was used. For the data collection, the instrument used, based on the literature, enabled us to measure the learning styles. The data were analyzed using descriptive statistics and the Wilcoxon test. The sample included 111 students enrolled in the second half of 2015 and the first half of 2016, representing $88 \%$ of the study population. The results indicated a predominance of active, sensorial, visual and sequential learning styles. In relation to academic achievement, higher averages were achieved in the individual evaluations (summations). In the comparison of performance and the students' learning styles, a balance was identified across the dimensions, i.e. the averages are close both for the individual and group evaluations, as well as for the half-year and the overall Academic Performance Coefficient (APC). Wilcoxon's nonparametric test indicated no difference in most of the results, except for the summative and sequential/global style evaluations.

Keywords: Learning Styles. Academic performance. Accounting Sciences.

\section{RESUMEN}

El objetivo del estudio fue verificar si el estilo de aprendizaje impacta en el rendimiento académico de los alumnos que cursaron la materia de Análisis de Costos en la carrera de Ciencias Contables en lo que se refiere a las evaluaciones formativas y sumativas. Oportunamente, se compararon la asociación del rendimiento en la referida materia y el rendimiento del alumno en el semestre, como también el coeficiente general de la carrera. Para el análisis del rendimiento académico, se utilizó la Teoría de la Evaluación. Para la recolección de datos, el instrumento utilizado, basado en la literatura, posibilito medir el estilo de aprendizaje. Para el análisis de los datos, se utilizaron la estadística descriptiva y la prueba de Wilcoxon. La muestra comprende 111 alumnos matriculados en el segundo semestre del año de 2015 y el primer semestre de 2016, representando 88\% de la población. Los resultados apuntan el predominio de los estilos activo, sensorial, visual y secuencial. Cuanto al rendimiento académico, se verificaron mayores promedios para las evaluaciones individuales (sumativas). En comparación al rendimiento y a los estilos de aprendizaje de los alumnos, se identificó un equilibrio en las dimensiones, o sea, los promedios son próximos tanto para los individuales como en grupo y, también, los promedios del Coeficiente de Rendimiento Académico (CRA) semestral y general. La prueba no paramétrico Wilcoxon indico que no hay diferencia en la mayoría de los resultados, con excepción entre la evaluación sumativa y el estilo secuencial/global.

Palabras clave: Estilos de Aprendizaje. Rendimiento Académico. Ciencias Contables.

\section{INTRODUÇÃO}

A busca por mais eficiência no ensino tem contribuído para o desenvolvimento de pesquisas sobre estilos de aprendizagem (Albuquerque, Nunes, Batista, Luz \& Carvalho, 2015), encontrando-se os estudiosos dessa temática empreendendo esforços para evidenciar como indivíduos interagem em ambientes de aprendizagem (Silva \& Oliveira, 2010). Esses estilos podem ser entendidos, de forma breve, como um agrupamento das características comportamentais de um indivíduo e estando alinhados com os métodos de ensino adotados poderão resultar em um melhor desempenho acadêmico (Cerqueira, 2000; Silva, 2006).

A identificação dos estilos de aprendizagem e o seu uso em prol dos objetivos educacionais são, segundo Silva e Oliveira (2010), pertinentes para docentes e discentes. Para os docentes, isso é importante, pois poderão desenvolver seus métodos de ensino, buscando contemplar os diferentes estilos identificados nas salas de aulas, 
bem como para os discentes, os quais poderão buscar meios de se adaptarem quando desenvolverem atividades que não são favoráveis ao seu estilo de aprendizagem.

Em consonância, Cordeiro e Silva (2012) afirmam que o conhecimento sobre os estilos de aprendizagem oportuniza a adequação do ensino com vistas a atender aos alunos, o que pode melhorar a qualidade do processo de ensino-aprendizagem. Albuquerque et al. (2015) destacam ainda que, ao considerar tais estilos, é possível aprimorar o ensino e promover maior assimilação do conhecimento. Nesse sentido, estudos anteriores apontam a importância de se investigarem os estilos dos alunos, bem como a relação desses com o desempenho acadêmico (Silva \& Oliveira, 2010; Silva, Leal, Pereira \& Oliveira, 2015; Meurer, Pedersini, Antonelli \& Voese, 2017).

No que se refere ao desempenho acadêmico, considera-se que esse representa o resultado auferido no processo de avaliação, podendo ser mensurado de diversas formas: notas de avaliações, disciplinas, conceitos, médias, entre outros (Munhoz, 2004; Miranda, Lemos, Oliveira \& Ferreira, 2015). Sob a luz da Teoria da Avaliação, Shaftel e Shaftel (2007) asseveram que a avaliação é utilizada para três propósitos principais: informar aos alunos sobre o desempenho auferido, prestar contas aos interessados (gestores educacionais, governo, entre outros) e ainda à própria instituição, a qual poderá desenvolver maneiras de melhorar ou manter o desempenho.

Para a análise do rendimento acadêmico dos alunos em uma determinada disciplina, são utilizadas várias formas de avaliações, sendo elas: individuais, em grupo, questões objetivas e/ou subjetivas, estudos de caso, entre outras que ocorrem durante o processo de ensino, denominadas avaliações formativas, podendo ocorrerem, ainda, após o processo, as avaliações somativas (Shaftel \& Shaftel, 2007).

Diante do exposto, pretende-se investigar o seguinte problema de pesquisa: qual relação entre os estilos de aprendizagem e o rendimento acadêmico de estudantes de contabilidade na disciplina Análise de Custos?

Assim, a presente pesquisa tem o objetivo de verificar se o estilo de aprendizagem impacta o rendimento acadêmico nas avaliações formativas e somativas dos alunos que cursaram a disciplina Análise de Custos no curso de graduação em Ciências Contábeis. Para isso, foram mapeados os estilos dos alunos que cursaram a disciplina Análise de Custos no curso de Ciências Contábeis de uma IES pública federal localizada no Estado de Minas Gerais, utilizando-se o instrumento desenvolvido por Felder e Soloman (1991). Posteriormente, realizaramse os testes estatísticos, com o intuito de verificar o impacto dos estilos no rendimento acadêmico auferido na disciplina, considerando as avaliações formativas e somativas, bem como verificar o impacto no Coeficiente de Rendimento Acadêmico (CRA) semestral e geral dos discentes.

Deve-se ressaltar a relevância das disciplinas na área de custos no âmbito do curso de Ciências Contábeis, o que pode ser verificado no estudo de Faria e Leal (2014). As autoras pesquisaram as instituições que oferecem o curso no Estado de Minas Gerais e identificaram, por meio das grades curriculares, que todas as instituições investigadas ofereciam disciplinas relacionadas à contabilidade gerencial, abrangendo a temática de custos. Evidencia-se, portanto, que as disciplinas relacionadas a custos apresentam conteúdos relevantes ao exercício profissional, especificamente a disciplina Análise de Custos, a qual está voltada à utilização das informações de custos para embasar o processo decisório, despertando nos alunos um perfil analítico que é demandado pelo mercado.

Ainda em relação ao estudo sobre contabilidade gerencial, destaca-se a pesquisa de Borges, Santos, Abbas, Marques e Tonin (2014), a qual evidenciou uma média de $42 \%$ de reprovação na disciplina de Contabilidade de Custos, no período de 2008 a 2013, no que tange aos discentes do curso de Contabilidade na Universidade Estadual de Maringá. Dessa forma, percebe-se a relevância de se conhecer o comportamento dos discentes que cursam disciplinas relacionadas a custos, uma vez que esse é um componente em destaque nas grades curriculares e ainda apresenta um baixo índice de aprovação, o que pode contribuir para a evasão no curso de Ciências Contábeis.

O presente estudo contribuirá com os docentes, revelando a eles a importância do alinhamento entre 0 ensino e os estilos de aprendizagem presentes, o que poderá contribuir para uma melhoria no processo ensinoaprendizagem. Dessa forma, espera-se que os docentes levem em consideração a individualidade no momento do ensino. Além disso, espera-se que um processo de ensino alinhado entre professor-aluno tenha maior eficácia, podendo contribuir com a diminuição da evasão escolar, tendo em vista uma melhor adaptação dos alunos no processo.

Espera-se também que a presente pesquisa contribua para os estudos no campo de educação contábil, visto que ela irá apresentar um perfil para os discentes da área quanto aos estilos de aprendizagem predominantes e sua relação com o rendimento auferido. 
Para alcançar o objetivo, apresenta-se na próxima seção a revisão da literatura, que serviu de base teórica para o desenvolvimento desta pesquisa. Na sequência, evidenciam-se os métodos utilizados e posteriormente os resultados encontrados. Na última seção, apresentam-se as considerações finais.

\section{REVISÃO DA LITERATURA}

Para evidenciar a fundamentação teórica desta pesquisa, apresentam-se conceitos e estudos anteriores sobre os estilos de aprendizagem. E na sequência, conceitos relativos ao desempenho acadêmico, bem como a forma de avaliação utilizada no ambiente acadêmico, com foco no ensino superior.

\subsection{Estilos de Aprendizagem}

As pesquisas sobre estilos de aprendizagem têm sido realizadas em diversas áreas do conhecimento, fornecendo diferentes conceitos e modelos sobre o assunto (Silva, 2012). Contudo, é percebido que muitos autores associam tais conceitos a características no processo de aprendizagem (Silva \& Oliveira, 2010) e apresentam como aspecto em comum a concepção da existência de diferenças individuais na aprendizagem, ou seja, as pessoas apresentam particularidades no modo de aprender (Silva, 2012).

Considerando que os estudantes aprendem de formas diferentes, Felder e Silverman (1988) compreendem a aprendizagem como um processo de duas etapas, as quais abrangem o recebimento e 0 processamento de informações. Os autores entendem que as informações ficam disponíveis para que os alunos as recebam por meios de seus sentidos ou são geradas internamente e, em um segundo momento, elas passam por um processamento, por exemplo, por memorização ou raciocínio, assim o aluno aprende ou não determinado conteúdo. Nessa perspectiva, os autores defendem que os estilos de aprendizagem compreendem algumas dimensões, evidenciando a forma como o aluno recebe e processa uma informação.

Silva (2006) afirma que os estilos de aprendizagem estão associados às particularidades no que tange à aquisição do conhecimento, assumindo que há diferentes formas de aprender e que essas refletem no processo de aprendizagem. A autora destaca, ainda, que o desenvolvimento dos estudos sobre os estilos de aprendizagem promoveu a criação de modelos que possibilitam a mensuração dos estilos dos estudantes.

Por sua vez, Silva et al. (2015) destacam que os principais modelos teóricos que são utilizados como base para os instrumentos de avaliação dos estilos de aprendizagem são o de Myers-Briggs - MBTI (1970), o Modelo de Kolb - LSI (1984), e o Modelo de Felder e Silverman - ILS (1988). Neste estudo, será utilizado o instrumento desenvolvido por Felder e Soloman (1991), o qual se baseia no instrumento de Felder e Silverman (1988).

De acordo com Felder e Silverman (1988), a partir das respostas a cinco questões centrais, seria possível entender 0 estilo de aprendizagem do discente, havendo, para cada pergunta, dois tipos de respostas, conforme apresentado no Figura 1.

\begin{tabular}{|l|l|}
\hline Pergunta & Respostas \\
\hline $\begin{array}{l}\text { 1) Qual informação é percebida } \\
\text { preferencialmente pelo estudante? }\end{array}$ & $\begin{array}{l}\text { Sensorial (externa) - imagens, sons, sensações físicas, etc. } \\
\text { Intuitiva (interna) - possibilidades, intuição, dicas, etc. }\end{array}$ \\
\hline $\begin{array}{l}\text { 2) Por meio de qual canal sensorial a informação } \\
\text { externa é recebida mais eficientemente? }\end{array}$ & $\begin{array}{l}\text { Visual - diagramas, gráficos, desenhos, etc. } \\
\text { Auditivo - palavras e sons }\end{array}$ \\
\hline $\begin{array}{l}\text { 3) Com qual organização da informação o } \\
\text { estudante se sente mais confortável? }\end{array}$ & $\begin{array}{l}\text { Indutiva - fatos e observações são dados e princípios são inferidos } \\
\text { Dedutiva - os princípios são apresentados e as consequências são } \\
\text { deduzidas }\end{array}$ \\
\hline $\begin{array}{l}\text { 4) Como o estudante prefere processar a a a } \\
\text { informação? }\end{array}$ & $\begin{array}{l}\text { Ativamente - por meio do envolvimento direto em atividades físicas ou } \\
\text { em discussões } \\
\text { Reflexivamente - de um modo mais introspectivo }\end{array}$ \\
\hline 5) Como o estudante estrutura a informação? & $\begin{array}{l}\text { Sequencialmente - utilizando-se de uma sequência de passos (linear) } \\
\text { Globalmente - por meio de mapas mentais e elos (não lineares) }\end{array}$ \\
\hline
\end{tabular}

Figura 1. Modelo de Felder e Silverman baseado em cinco perguntas básicas Fonte: Adaptada de Felder e Silverman (1988, p.675). 
Assim, para cada dimensão, percepção, entrada (também chamada de retenção), organização, processamento e compreensão, existem dois estilos de aprendizagem, são eles: sensorial $\mathrm{x}$ intuitivo, visual $\mathrm{x}$ auditivo (que, posteriormente, se tornou visual $x$ verbal), indutivo $x$ dedutivo, ativo $x$ reflexivo e sequencial $x$ global, os quais irão apresentar as características preferenciais do indivíduo para aprender. A diferença entre o ILS de Felder e Silverman (1988) e o de Felder e Soloman (1991) consiste na exclusão da dimensão organização.

Os estilos de aprendizagem revelam algumas características sobre os sujeitos. Os indivíduos com estilo sensorial, por exemplo, são um pouco mais lentos que os de estilo intuitivo que, por sua vez, são mais criativos na resolução de problemas. Os alunos com estilo ativo se adaptam bem a estudos em grupo e dinâmicas; enquanto que os reflexivos preferem estudos individualizados. Já os que apresentam estilo sequencial conseguem trabalhar com uma sequência lógica de etapas, mas conseguem assimilar apenas informações fragmentadas e superficiais, enquanto que os de estilo global precisam de uma visão geral do assunto para facilitar 0 aprendizado (Felder \& Silverman, 1988).

Felder e Silverman (1988) asseveram que o indivíduo aprende por um estilo ou por outro, não sendo capaz de compreender pelos dois estilos em uma dimensão. Dessa forma, existem os níveis de predominância dos estilos, podendo ser leve, moderada ou forte.

Silva et al. (2015) destacam a confiabilidade e a validade do instrumento proposto por Felder e Silverman (1988), além da acessibilidade, visto que está disponível em diversos idiomas. Diante disso, apresentam-se alguns estudos sobre estilos de aprendizagem (Silva \& Oliveira, 2010; Silva, 2012; Silva et al., 2015; Souza, Avelino \& Takamatsu, 2017), que utilizaram o ILS de Felder e Soloman (1991).

Silva e Oliveira (2010) e Silva (2012) verificaram os estilos de aprendizagem dos alunos de contabilidade em universidades diferentes, porém ambas no Estado de São Paulo. No primeiro estudo, constatou-se que os estilos predominantes foram sensorial e visual, já no segundo, a predominância foi sensorial e sequencial, seguida pelos estilos ativo e visual.

Silva et al. (2015) analisaram os estilos de aprendizagem de alunos de três cursos de especialização a distância na área de administração pública, sendo eles Gestão Pública, Gestão Pública Municipal e Gestão Pública em Saúde. Em seus resultados, os autores constataram que os estilos predominantes foram ativo, sensorial, verbal e sequencial.

De forma similar, Borges (2016) e Souza et al. (2017) pesquisaram sobre os estilos de aprendizagem de graduandos em Ciências Contábeis em diferentes instituições de ensino no Estado de Minas Gerais. Em ambas as pesquisas, os estilos predominantes dos estudantes foram ativo, visual, sensorial e sequencial.

Em suma, os estudos citados evidenciam como os alunos apresentam formas diferentes de aprender, 0 que, consequentemente, gera reflexos dentro das salas de aula. Nesse sentido, Silva (2006) aponta que conhecer os estilos de aprendizagem presentes em uma sala de aula é de fundamental importância para o professor, pois este poderá utilizar em suas aulas um número maior de técnicas de ensino que atenda às preferências de aprender dos discentes, bem como motivar os alunos no processo.

Diante do exposto, entende-se que verificar possíveis impactos que os estilos de aprendizagem exercem no desempenho acadêmico dos discentes se torna de grande importância. Para tanto, no próximo tópico, são abordados os conceitos no que tange ao desempenho acadêmico, bem como as formas de avaliação que podem ser empregadas, além de alguns estudos anteriores sobre a temática.

\subsection{Desempenho acadêmico e Teoria da Avaliação}

O desempenho acadêmico, segundo Munhoz (2004), pode ser definido como o retorno obtido do desempenho das tarefas acadêmicas, que representa o nível de habilidade alcançado pelo estudante. 0 autor ainda complementa que o "termo desempenho envolve a dimensão da ação e o rendimento é o resultado de sua avaliação, expresso na forma de notas ou conceitos obtidos" (Munhoz, 2004, p.52). Contudo, tais conceitos são frequentemente utilizados com o mesmo significado.

Existem várias formas utilizadas nas instituições de ensino superior para medir o desempenho dos discentes. Miranda et al. (2015) destacam medidas internas, tais como notas em avaliações em disciplinas e média geral acumulada (com ou sem ajustes), bem como exames externos à instituição, como o Exame Nacional de Desempenho dos Estudantes (ENADE). 
O desempenho acadêmico pode ser influenciado por variáveis relacionadas ao corpo docente, discente e à instituição de ensino (Corbucci, 2007). Destaca-se, porém, que as variáveis relacionadas aos discentes são indicadas como as que exercem maior influência no desempenho dos mesmos (Ferreira et al., 2002, Souza, 2008; Santos, 2012).

Diante disso, o presente trabalho discute sobre os estilos de aprendizagem dos alunos como uma variável que pode afetar o desempenho acadêmico, evidenciando-se, a seguir, alguns estudos que investigaram essa relação. Ressalta-se que tais estudos utilizaram diversos instrumentos, sendo os principais os de Kolb (1984), de Felder e Silverman (1988) e de Felder e Soloman (1991).

Cordeiro e Silva (2012) identificaram que o desempenho dos alunos em disciplinas de Administração Financeira independe dos estilos de aprendizagem presentes na amostra. Nogueira, Espejo, Reis \& Voese (2012) auferiram o mesmo resultado, porém, nesse caso, foram observados estudantes de Administração na modalidade EaD nas disciplinas Contabilidade Geral e Gerencial. Pellón, Nome e Arán (2013) também constataram, em estudo com discentes do quinto ano de Medicina, que os estilos atuam com independência.

Por outro lado, Dias, Sauaia e Yoshizak (2013) constataram diferença significativa entre o desempenho e os estilos de aprendizagem ativo-reflexivo e visual-verbal em estudo com alunos de dois cursos de especialização, logística empresarial e gestão de operações. Já Silva e Oliveira (2010) investigaram alunos de contabilidade e observaram que os discentes com estilo intuitivo, verbal e global são os que auferem melhores resultados.

Meurer et al. (2017) também pesquisaram os estilos de aprendizagem em graduandos de Ciências Contábeis e verificaram relação do estilo com rendimento acadêmico quando os estilos dos alunos coincidem com o do professor. A partir desse resultado, os autores perceberam que os alunos com o mesmo estilo do professor apresentaram menor índice de reprovação.

Considerando que as medidas de rendimento dos alunos são obtidas a partir de processos avaliativos, conforme evidenciado por Miranda et al. (2015), apresentam-se a seguir aspectos relativos à avaliação educacional. Ressalta-se que o estudo dessa temática surgiu na década de 40, principalmente, com o trabalho de Raiph W. Tyler, e se desenvolveu na década de 60 com os trabalhos de Lee J. Cronbach, Michael Scriven e Robert E. Stake (Vianna, 1982).

Para Tyler (1942), a avaliação tem diversas finalidades, dentre elas, oferecer um feedback, apontando os pontos de melhorias no processo de ensino e aprendizagem, orientação básica para desenvolvimento efetivo dos alunos, entre outras. Percebe-se, assim, que Tyler (1942) defendia a avaliação não somente para medir características individuais, mas também a necessidade de se atentar ao currículo e seus objetivos. Já Cronbach (1963) se diferenciou ao apontar o uso da avaliação para tomada de decisões (Gonçalves, 2005).

Por sua vez, Scriven (1967, como citado em Vianna, 1982, p.11) apresenta a avaliação "como um levantamento sistemático de informações e sua posterior análise para fins de determinar o valor de um fenômeno educacional". Stake (1967) ressalta a complexidade do processo educacional pelo qual a avaliação deve se orientar. Evidencia-se que os autores mencionados serviram de base para a concepção da avaliação moderna, que foi se modificando paralelamente ao contexto econômico e político (Gonçalves, 2005).

Vianna (1982) aponta que a avaliação foi entendida, por muito tempo, apenas como a mensuração do desempenho escolar, sendo utilizada, segundo Tyler (1942), para a classificação dos alunos, agrupamentos em classe, relatórios aos pais, relatórios financeiros e outros. No entanto, a avaliação é uma atividade muito mais ampla, pois abrange 0 aluno e suas dificuldades de aprendizagem, abrangendo também o grupo, bem como os programas e materiais utilizados, devendo-se, ainda, avaliar todo o sistema educacional (Viana, 1982).

Dessa forma, a avaliação pode também ser compreendida como instrumento a ser utilizado pelo professor, com o propósito de conseguir informações sobre o aprendizado dos alunos, obtendo, assim, base para que 0 próprio professor possa empreender ações que auxiliem os alunos na continuidade de seus estudos (Cavalcanti \& Aquino, 2009).

Portanto, a avaliação é utilizada com três objetivos centrais: informar os alunos sobre o desempenho auferido, prestar contas aos interessados (gestores educacionais, governo, entre outros, que irão embasar suas estratégias a partir dos dados coletados) e, ainda, à própria instituição, que poderá desenvolver maneiras de melhorar ou manter o seu desempenho (Shaftel \& Shaftel, 2007). 
Santos (2005) destaca que, para o sucesso do processo de ensino-aprendizagem, a avaliação deve ser dividida em três funções: a diagnóstica, a formativa e a somativa. Shaftel e Shaftel (2007) apontam apenas duas funções da avaliação, a formativa e a somativa.

A avaliação diagnóstica, de acordo com Santos (2005), seria aquela que identifica o conhecimento inicial do aluno frente àquele conteúdo. Esse tipo de avaliação deve ocorrer no início, observando-se as deficiências existentes e as possíveis causas. Um exame de bolsas para uma escola de ensino médio ou de línguas seria bom exemplo dessa forma de avaliação.

Já a avaliação formativa está relacionada aos esforços realizados para complementar o aprendizado e ocorre ao longo do processo de ensino-aprendizagem (Shaftel \& Shaftel, 2007). Esse tipo de avaliação funciona como um termômetro, pois, ao longo do processo, apresenta os erros e os acertos cometidos pelos alunos e professores, os quais podem ser corrigidos a tempo (Santos, 2005). As atividades, as provas e os testes aplicados no decorrer do curso podem ser considerados como exemplos dessa categoria.

Por fim, a avaliação somativa acontece após o módulo de aprendizagem e tem o intuito de classificar os alunos de acordo com o seu aproveitamento, com o objetivo de elevação de posição (Shaftel \& Shaftel, 2007; Santos, 2005). Como exemplos, podem ser citados um vestibular, um exame de ordem ou até mesmo um concurso público. Santos (2005) ainda destaca que a avaliação somativa pode acontecer no fim de um módulo de curso, de uma disciplina, de um semestre, entre outros.

Diante disso, pode-se inferir que a avaliação diagnóstica acontece antes, a formativa ocorre durante e a somativa se dá depois do processo de aprendizagem, sendo as duas primeiras, normalmente, desenvolvidas internamente, ou seja, na instituição de ensino, e a última é desenvolvida por órgãos externos. No caso desta pesquisa, o foco são as avaliações formativas e somativas desenvolvidas na disciplina Análise de Custos.

Shaftel e Shaftel (2007) apontam que, com o intuito de medir o desempenho acadêmico, tem-se ainda a divisão da avaliação em direta, aquela que acontece por meio de testes; e a avaliação indireta, que ocorre por meio da realização de pesquisas. Nas avaliações diretas, são utilizados os testes de desempenho, objetivos e subjetivos, sendo os primeiros caracterizados por disporem de apenas uma resposta correta, facilitando a forma de correção do professor, enquanto que os subjetivos necessitam de uma avaliação mais subjetiva, visto a complexidade das respostas ou das atividades compreendidas (Shaftel \& Shaftel, 2007).

Considerando as diversas formas de avaliação, Garcia (2009) ressalta a relevância do professor, afirmando que as práticas utilizadas pelo docente podem interferir no desenvolvimento dos alunos. Contudo, o autor reconhece que, tradicionalmente, muitas práticas avaliativas utilizadas no ensino superior se deparam com algumas limitações e a maior parte dessas ocorrem na avaliação somativa.

Entende-se assim que as escolhas do professor no que tange ao processo avaliativo reflete nas "atitudes de aprendizagem" dos alunos, na forma como esses estudam e no significado que dão às atividades acadêmicas. Logo, torna-se evidente a necessidade da reflexão por parte dos docentes sobre a forma como conduzem 0 processo avaliativo no ensino, visto a influência desse processo na aprendizagem dos estudantes (Garcia, 2009).

Nesse sentido, Cavalcanti e Aquino (2009) destacam que a reflexão acerca do processo de avaliação está relacionada a um conjunto de decisões que visam à melhoria do ensino e da aprendizagem dos estudantes, tornando-se, portanto, essencial que se reflita sobre os objetivos pretendidos com a avaliação.

Diante da relevância do processo avaliativo no ensino e, consequentemente, no desempenho dos alunos, o presente trabalho verifica a relação entre estilos de aprendizagem e rendimento nas avaliações formativa e somativa. Compreende-se que, ao considerar os estilos de aprendizagem na escolha das atividades avaliativas, o professor contempla mais amplamente as diferentes formas de aprender de seus alunos, o que pode refletir nos resultados por eles alcançados. 


\section{ASPECTOS METODOLÓGICOS}

Esta pesquisa, quanto aos objetivos, caracteriza-se como descritiva, pois tem o intuito de descrever as características da amostra em estudo no que diz respeito aos estilos de aprendizagem e à relação com 0 rendimento acadêmico na disciplina Análise de Custos oferecida no curso de Ciências Contábeis. Quanto à abordagem do problema, a mesma é de cunho quantitativo, pois irá utilizar testes estatísticos para o tratamento dos dados. Já em relação aos procedimentos de coleta de dados, a mesma é do tipo levantamento, utilizando-se de um questionário e também da pesquisa documental, com o fim de identificar o rendimento acadêmico dos participantes da pesquisa.

0 instrumento de coleta de dados foi organizado em dois blocos de perguntas: um direcionado à caracterização do respondente, incluindo o gênero, a idade, a experiência profissional, o turno, além de solicitar o número de matrícula para que fosse possível a obtenção do rendimento auferido na disciplina Análise de Custos; e a segunda parte foi composta pelo ILS de Felder e Soloman (1991), contendo questões que determinam os estilos de aprendizagem dos discentes.

O ILS de Felder e Soloman é composto por 44 questões, sendo essas divididas em quatro dimensões: percepção, retenção, processamento e compreensão. Cada dimensão possui 11 questões, as quais apresentam duas alternativas de resposta, $A$ ou $B$. Após a categorização das respostas, foi realizado o cálculo que determina o estilo do respondente em cada uma das dimensões, conforme recomenda Lopes (2002).

A pesquisa foi realizada com discentes do curso de Ciências Contábeis em uma instituição pública federal, localizada no Estado de Minas Gerais, matriculados na disciplina Análise de Custos, que é ofertada em dois turnos (integral e noturno), sendo oferecida no $5^{\circ}$ período do curso. Destaca-se que a escolha por tal instituição se deu por acessibilidade e a escolha pela disciplina se justifica por evidências da literatura que apontam a relevância desse conteúdo curricular no curso de Ciências Contábeis (Faria \& Leal, 2014), bem como o baixo desempenho dos alunos na área que abrange tal disciplina (Borges et al., 2014).

Assim sendo, a população do estudo compreende duas turmas, com um total de 127 alunos matriculados na referida disciplina; e a amostra obtida corresponde a 111 alunos, que responderam o questionário e concordaram em participar da pesquisa, o que representa $88 \%$ da população.

A coleta de dados ocorreu nos meses de dezembro/2015 e julho/2016. O questionário foi aplicado em sala de aula com a autorização do docente responsável pela disciplina Análise de Custos. Antes da aplicação do questionário, foi explicado pelos pesquisadores o objetivo da pesquisa, bem como o instrumento. Cabe ressaltar que, no cabeçalho do questionário, foi apresentado o Termo de Consentimento Livre e Esclarecido.

Para complementar a coleta de dados, utilizou-se a pesquisa documental, representada por uma planilha de notas da disciplina em estudo. As notas estavam separadas por avaliações feitas individualmente pelos alunos e avaliações realizadas em grupo. A planilha de notas foi disponibilizada pelo docente responsável pela disciplina. Verificou-se, junto à Coordenação de Curso, o coeficiente de rendimento acadêmico (CRA) dos alunos participantes da pesquisa, tendo sido disponibilizado um relatório pelo número de matrícula, constando o CRA dos semestres analisados e o CRA geral de cada aluno. A instituição em estudo utiliza o cálculo do CRA, que é realizado ao final de cada período letivo (semestre), considerando três casas decimais e cumulativamente em relação aos períodos anteriores. A fórmula apresentada para cálculo do CRA é a seguinte:

Onde:

$$
C R A=\frac{\sum\left(\operatorname{Nota} x C_{c}\right)}{\sum C H_{m}} x\left(1-\frac{1}{2} \frac{\sum C H_{r f}}{\sum C H_{m}}\right)
$$

$\mathrm{CH}_{c}$ : carga horária cursada (componentes curriculares cursados com aprovação e componentes curriculares cursados com reprovação);

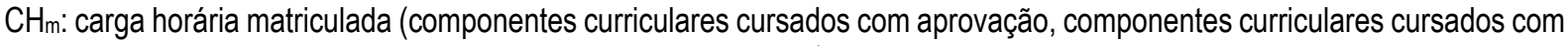
reprovação e componentes curriculares com trancamento parcial);

$\mathrm{CH}_{\mathrm{rf}}$ : carga horária em componentes curriculares com reprovação por frequência.

Para a medida de desempenho acadêmico, utilizou-se o rendimento (nota) auferido na disciplina Análise de Custos, classificado de acordo com a Teoria da Avaliação e, ainda, o CRA geral e semestral dos alunos. Para classificar as avaliações conforme a Teoria da Avaliação (Shaftel \& Shaftel, 2007), verificou-se com o docente responsável pela disciplina como as atividades foram dispostas no decorrer do semestre. 0 referido docente 
informou que foram aplicadas avaliações individuais (duas provas) e em grupo (estudo de caso, debates, repórter da semana com reportagens sobre temas da disciplina, resenha, trabalho de vídeo aula e exercícios avaliativos).

A partir de tais informações e considerando a Teoria da Avaliação, foi possível classificar as avaliações em formativas, que foram aplicadas com o intuito de acompanhar a evolução dos discentes durante um processo de aprendizagem, e avaliações somativas, que foram aplicadas ao fim da oferta do conteúdo. A Figura 2 ilustra 0 processo de avaliação adotado na disciplina em estudo.

\begin{tabular}{|c|c|c|}
\hline \multicolumn{2}{|l|}{ Teoria da Avaliação } & Avaliação utilizada na disciplina \\
\hline \multirow{2}{*}{ Funções da avaliação } & Formativa & Atividades desenvolvidas em grupo \\
\hline & Somativa & Atividades individuais \\
\hline \multirow{2}{*}{$\begin{array}{c}\text { Quando ocorre o processo } \\
\text { avaliativo }\end{array}$} & \multicolumn{2}{|c|}{ Durante o semestre, ao longo do processo de ensino-aprendizagem } \\
\hline & \multicolumn{2}{|r|}{ Após finalizar o conteúdo programático proposto } \\
\hline \multirow[t]{2}{*}{ Tipos de Avaliação } & Indireta & $\begin{array}{l}\text { Estudos de caso, atividades em grupo, trabalho vídeo aula, repórter da } \\
\text { semana, resenhas, exercícios avaliativos }\end{array}$ \\
\hline & Direta & Testes no Moodle e prova \\
\hline
\end{tabular}

Figura 2: Relação das atividades da disciplina com a Teoria da Avaliação Fonte: Elaborado pelos autores.

Para tratamento dos dados, utilizou-se, primeiramente, a estatística descritiva com o fim de demonstrar a caracterização dos respondentes, os estilos de aprendizagem predominantes, bem como o desempenho acadêmico dos discentes na disciplina analisada e, ainda, o comportamento desse desempenho com o CRA geral e semestral por eles alcançado.

Já com a intenção de verificar se o estilo de aprendizagem impacta no rendimento acadêmico nas avaliações formativas e somativas dos alunos que cursaram a disciplina Análise de Custos no curso de Ciências Contábeis, bem como a relação do estilo com o coeficiente de rendimento acadêmico, utilizou-se o teste de Wilcoxon.

O referido teste é utilizado para comparar as medianas de duas amostras emparelhadas, analisando, assim, a direção das diferenças entre e dentro dos pares. Esse teste é classificado como não paramétrico, sendo adotado quando não há normalidade dos dados (Fávero, Belfiore, Silva \& Chan, 2009). Tais características justificam seu uso no presente estudo, tendo sido consideradas as seguintes hipóteses:

$\mathrm{H}_{0}$ : $\mathrm{O}$ desempenho acadêmico dos estudantes de Ciências Contábeis na disciplina Análise de Custos independe dos estilos de aprendizagem dos alunos;

$\mathbf{H}_{1}$ : O desempenho acadêmico dos estudantes de Ciências Contábeis na disciplina Análise de Custos tem relação de dependência com os estilos de aprendizagem dos alunos;

A análise buscou verificar a dependência do desempenho acadêmico em relação à dimensão dos estilos de aprendizagem, ou seja, o teste utilizado identificou se o rendimento apresentava diferenças significativas, dependendo do estilo de aprendizagem. Ressalta-se que a análise foi realizada de forma segregada, considerando quatro grupos: rendimento nas avaliações somativas (individuais); rendimento nas avaliações formativas (em grupo); CRA semestral; e CRA geral dos alunos. Essas quatro medidas do rendimento acadêmico foram consideradas como variáveis dependentes e os estilos de aprendizagem obtidos pelo ILS de Felder e Soloman (1991) como as variáveis independentes.

\section{ANÁLISE E DISCUSSÃO DOS RESULTADOS}

Inicialmente, são apresentados, na Tabela 1, os resultados referentes à caracterização da amostra, bem como as informações dos participantes da pesquisa. 
Tabela 1

Perfil dos respondentes

\begin{tabular}{|c|c|c|c|}
\hline Sexo & & Turno Matriculado & \\
\hline Feminino & $59,50 \%$ & Integral & $36 \%$ \\
\hline Masculino & $40,50 \%$ & Noturno & $64 \%$ \\
\hline Idade & & Cursou Ensino Médio & \\
\hline Até 20 anos & $49,50 \%$ & Todo em escola pública & $80,20 \%$ \\
\hline Entre 21 a 25 anos & $41,40 \%$ & Todo em escola particular & $16,20 \%$ \\
\hline Acima de 25anos & $9,00 \%$ & Maior parte em escola pública & $1,80 \%$ \\
\hline & & Maior parte em escola particular & $1,80 \%$ \\
\hline \multicolumn{4}{|c|}{ Experiência Profissional } \\
\hline Sim (não foi na área) & $38,50 \%$ & & \\
\hline Sim (na área) & $45,90 \%$ & & \\
\hline Não tenho experiência & $15,60 \%$ & & \\
\hline
\end{tabular}

Fonte: Elaborada a partir dos dados da pesquisa.

O perfil da amostra é caracterizado por uma maioria de estudantes do sexo feminino matriculada no turno integral e com faixa etária de até 20 anos. Além disso, houve uma predominância de discentes que cursaram todo o ensino médio em escola pública. Verificou-se, ainda, que um percentual relevante $(84,4 \%)$ tem experiência profissional, sendo $45,9 \%$ na área contábil. Na sequência, apresentam-se os resultados identificados com a análise descritiva dos dados.

\subsection{Análise Descritiva e Teste de Wilcoxon}

Com a análise dos dados da pesquisa, é possivel constatar que o perfil dos alunos, no que se refere aos estilos de aprendizagem, é caracterizado, predominantemente, como: ativo, sensorial, visual e sequencial. A Tabela 2 ilustra os resultados quanto aos estilos de aprendizagem.

Tabela 2

Distribuição da Amostra nas dimensões de estilos

\begin{tabular}{c|lcc}
\hline \multirow{4}{*}{} & \multicolumn{3}{|c}{ Dimensão Processamento } \\
\cline { 2 - 4 } & Ativo & 71 & $64,00 \%$ \\
& Reflexivo & 40 & $36,00 \%$ \\
\cline { 2 - 4 } Estilos de & \multicolumn{3}{c}{ Dimensão Percepção } \\
\cline { 2 - 4 } Aprendizagem & Sensorial & 89 & $80,20 \%$ \\
& Intuitivo & 22 & $19,80 \%$ \\
\cline { 2 - 4 } & \multicolumn{3}{|c}{ Dimensão Retenção } \\
\cline { 2 - 4 } & Visual & 96 & $13,50 \%$ \\
\cline { 2 - 4 } & Verbal & 15 & $65,80 \%$ \\
\cline { 2 - 4 } & & Dimensão Compreensão & $34,20 \%$ \\
\cline { 2 - 4 } & Sequencial & 73 & \\
& Global & 38 & \\
\end{tabular}

Fonte: Elaborada a partir dos dados da pesquisa.

Percebe-se que os estilos de aprendizagem revelam algumas características sobre os alunos investigados, quais sejam: os mesmos apresentam predominância do estilo ativo, indicando que se adaptam bem a estudos em grupo e dinâmicas (Felder \& Silverman, 1988). Com base na predominância desse estilo, espera-se que 0 desempenho nas atividades formativas seja maior.

$\mathrm{Na}$ dimensão compreensão, há maior predominância do estilo sequencial, pois tais estudantes conseguem trabalhar com uma sequência lógica de etapas e assimilar informações fragmentadas e superficiais (Felder \& Silverman, 1988).

Quanto à retenção, houve uma predominância do estilo visual, visto que demonstraram facilidade na aprendizagem por meio de imagens. Identificou-se, na dimensão percepção, um grande agrupamento de 
sensoriais, o que revela as características sistemáticas e objetivas dos alunos da área contábil (Silva \& Oliveira, 2010). $O$ estilo sequencial, na dimensão compreensão, apresentou um maior percentual, indicando que os alunos buscam informações e tomam decisões lineares. Felder e Silverman (1988) destacam que pessoas com o estilo sequencial seguem uma sequência lógica de etapas, conseguindo trabalhar com 0 ensino fragmentado ou superficial.

Os estilos com menor representatividade foram o verbal e intuitivo. Tais resultados são convergentes para os apontados por Silva e Oliveira (2010), Silva (2012) e Borges (2016), com estudos com discentes da área contábil. Além do mais, os estilos predominantes dos respondentes desta pesquisa são os mesmos de estudos anteriores (Silva \& Oliveira, 2010; Silva, 2012; Borges, 2016; Souza et al., 2017) também realizados com alunos de contábeis, revelando, quanto às características preferenciais, um perfil desse público propenso a aprender.

A análise descritiva do desempenho acadêmico (por avaliação e CRA), variável dependente, é demonstrada na Tabela 3. A nota individual é a soma das avaliações denominadas somativas realizadas individualmente pelos alunos. No caso da disciplina Análise de Custos, foram aplicadas duas provas e um teste no ambiente virtual de aprendizagem. Para as avaliações somativas, foram atribuídos 60 pontos. As notas em grupo se referem a atividades avaliativas, denominadas formativas, realizadas por grupos de 4 a 6 alunos, incluindo a resolução de estudos de caso, exercícios, vídeo aula e repórter da semana, aos quais foi atribuído 0 total de 40 pontos. O CRA representa o coeficiente de rendimento acadêmico, conforme descrito anteriormente, tendo sido informado pela coordenação de curso.

Tabela 3

Análise descritiva da variável dependente (rendimento acadêmico)

\begin{tabular}{ccccc}
\hline Rendimento/ Notas e CRA & Mínimo & Máximo & Média & Desvio padrão \\
\hline Individual & 1,00 & 75,00 & 38,77 & 14,62 \\
Grupo & 0,00 & 44,00 & 30,09 & 6,45 \\
Total & 8,00 & 100,00 & 68,85 & 16,26 \\
CRA semestral & 16,00 & 97,00 & 68,28 & 15,45 \\
CRA geral & 40,33 & 93,80 & 68,74 & 12,05 \\
\hline
\end{tabular}

Fonte: Elaborada a partir dos dados da pesquisa.

O rendimento dos alunos em estudo apresentou uma média menor nas avaliações realizadas em grupo (formativas) se comparadas com as avaliações individuais (somativas). Tal resultado se mostra contrário ao esperado, considerando que a maioria dos alunos apresentou estilo ativo, ou seja, eles têm preferências por atividades em grupo e por formas dinâmicas de aprendizagem.

$\mathrm{Na}$ Tabela 4, são apresentadas as médias do rendimento acadêmico, separando-se os alunos pelos estilos de aprendizagem.

Tabela 4

Média dos Rendimentos acadêmico da Amostra / Dimensão de Estilos

\begin{tabular}{|c|c|c|c|c|c|c|c|c|c|}
\hline \multicolumn{10}{|c|}{ Dimensão Processamento } \\
\hline \multicolumn{5}{|c|}{ Ativo } & \multicolumn{5}{|c|}{ Reflexivo } \\
\hline Notas & Mínimo & Máximo & Média & $\begin{array}{l}\text { Desvio } \\
\text { padrão }\end{array}$ & Notas & Mínimo & Máximo & Média & $\begin{array}{l}\text { Desvio } \\
\text { padrão }\end{array}$ \\
\hline Individual & 1,00 & 70,3 & 37,16 & 13,90 & Individual & 7,00 & 75,00 & 41,62 & 15,58 \\
\hline Grupo & 0,00 & 37,8 & 29,70 & 6,81 & Grupo & 17,00 & 44,00 & 30,78 & 5,76 \\
\hline $\begin{array}{l}\text { Total } \\
\text { CRA }\end{array}$ & 8,00 & 94,8 & 66,85 & 16,09 & $\begin{array}{l}\text { Total } \\
\text { CRA }\end{array}$ & 27,30 & 100,00 & 72,40 & 16,15 \\
\hline semestral & 16,00 & 85,6 & 66,78 & 16,19 & semestral & 36,00 & 97,00 & 70,74 & 13,87 \\
\hline CRA geral & 40,33 & 89,9 & 67,78 & 11,45 & CRA geral & 43,35 & 93,80 & 70,44 & 13,02 \\
\hline \multicolumn{10}{|c|}{ Dimensão Percepção } \\
\hline \multicolumn{5}{|c|}{ Sensorial } & \multicolumn{5}{|c|}{ Intuitivo } \\
\hline $\begin{array}{c}\text { Notas } \\
\text { Individual }\end{array}$ & $\begin{array}{c}\text { Mínimo } \\
1,00\end{array}$ & $\begin{array}{c}\text { Máximo } \\
70,50\end{array}$ & $\begin{array}{l}\text { Média } \\
39,05\end{array}$ & $\begin{array}{c}\text { Desvio } \\
\text { padrão } \\
14,84\end{array}$ & $\begin{array}{c}\text { Notas } \\
\text { Individual }\end{array}$ & $\begin{array}{c}\text { Mínimo } \\
8,00\end{array}$ & $\begin{array}{c}\text { Máximo } \\
75,00\end{array}$ & $\begin{array}{l}\text { Média } \\
35,85\end{array}$ & $\begin{array}{c}\text { Desvio } \\
\text { padrão } \\
14,56\end{array}$ \\
\hline
\end{tabular}


(Conclusão)

\begin{tabular}{|c|c|c|c|c|c|c|c|c|c|}
\hline \multicolumn{10}{|c|}{ Dimensão Percepção } \\
\hline \multicolumn{5}{|c|}{ Sensorial } & \multicolumn{5}{|c|}{ Intuitivo } \\
\hline Grupo & 0,00 & 44,00 & 30,27 & 6,69 & Grupo & 21,00 & 37,80 & 29,48 & 5,41 \\
\hline $\begin{array}{l}\text { Total } \\
\text { CRA }\end{array}$ & 8,00 & 96,00 & 69,32 & 16,80 & $\begin{array}{l}\text { Total } \\
\text { CRA }\end{array}$ & 60,00 & 100,00 & 78,67 & 20,13 \\
\hline semestral & 21,43 & 97,00 & 69,57 & 14,29 & semestral & 16,00 & 78,00 & 54,17 & 21,12 \\
\hline CRA geral & 42,14 & 93,80 & 69,39 & 11,91 & CRA geral & 40,33 & 77,10 & 59,66 & 10,49 \\
\hline \multicolumn{10}{|c|}{ Dimensão Retenção } \\
\hline \multicolumn{5}{|c|}{ Visual } & \multicolumn{5}{|c|}{ Verbal } \\
\hline Notas & Mínimo & Máximo & Média & $\begin{array}{l}\text { Desvio } \\
\text { padrão }\end{array}$ & Notas & Máximo & Média & \multicolumn{2}{|c|}{ Desvio padrão } \\
\hline Individual & 1,00 & 75,00 & 38,59 & 14,47 & Individual & 8,00 & 60,90 & 39,86 & 16,03 \\
\hline Grupo & 10,00 & 44,00 & 30,18 & 5,97 & Grupo & 0,00 & 37,30 & 29,50 & 9,17 \\
\hline $\begin{array}{l}\text { Total } \\
\text { CRA }\end{array}$ & 15,00 & 100,00 & 68,77 & 15,51 & $\begin{array}{l}\text { Total } \\
\text { CRA }\end{array}$ & 8,00 & 92,80 & 69,36 & 21,12 \\
\hline semestral & 16,00 & 97,00 & 67,78 & 15,36 & semestral & 21,43 & 86,80 & 70,92 & 16,30 \\
\hline CRA geral & 40,33 & 93,80 & 68,28 & 12,31 & CRA geral & 51,68 & 91,50 & 71,64 & 10,09 \\
\hline \multicolumn{10}{|c|}{ Dimensão Compreensão } \\
\hline \multicolumn{5}{|c|}{ Sequencial } & \multicolumn{5}{|c|}{ Global } \\
\hline Notas & Mínimo & Máximo & Média & $\begin{array}{l}\text { Desvio } \\
\text { padrão }\end{array}$ & Notas & Mínimo & Máximo & Média & $\begin{array}{l}\text { Desvio } \\
\text { padrão }\end{array}$ \\
\hline Individual & 1,00 & 75,00 & 36,81 & 14,95 & Individual & 9,00 & 70,50 & 42,52 & 13,34 \\
\hline Grupo & 0,00 & 44,00 & 30,12 & 7,01 & Grupo & 16,00 & 37,30 & 30,03 & 5,30 \\
\hline $\begin{array}{l}\text { Total } \\
\text { CRA }\end{array}$ & 8,00 & 100,00 & 66,92 & 17,65 & $\begin{array}{l}\text { Total } \\
\text { CRA }\end{array}$ & 35,20 & 95,50 & 72,55 & 12,60 \\
\hline semestral & 21,43 & 97,00 & 67,20 & 15,80 & semestral & 16,00 & 92,60 & 70,14 & 14,75 \\
\hline CRA geral & 40,33 & 93,80 & 67,55 & 12,56 & CRA geral & 44,31 & 87,64 & 71,01 & 10,80 \\
\hline
\end{tabular}

Fonte: Elaborada a partir dos dados da pesquisa.

Ao comparar as médias obtidas, tanto nas atividades da disciplina quanto no CRA semestral e geral, observou-se que a média dos discentes com estilo reflexivo (dimensão processamento) se mostrou sempre superior à média dos alunos com estilo ativo. Os discentes reflexivos são aqueles mais teóricos e preferem os estudos individuais (Felder \& Silverman, 1988). O mesmo ocorreu na dimensão compreensão, visto que os alunos com estilo global auferiram desempenho superior aos de estilo sequencial. Silva e Oliveira (2010) também constataram que os discentes com estilo global obtêm melhores resultados. Vale ressaltar que esse estilo revela a necessidade dos discentes em ter uma visão geral do conteúdo, bem como revela a facilidade de inter-relacionar conteúdos (Felder \& Silverman, 1988).

Para a dimensão percepção, as médias de discentes com estilo sensorial se mostraram superiores às daqueles discentes com perfil intuitivo, resultado divergente do encontrado por Silva e Oliveira (2010). Já no que tange à dimensão retenção, as médias dos discentes foram superiores às dos discentes com estilo verbal. Vale lembrar que, por ser um curso que exige um alto grau de leituras (legislações e normas), esperava-se que discentes com o estilo verbal que têm facilidade com tais atividades auferissem um resultado melhor, o que, de fato, foi comprovado.

Com 0 intuito de verificar se havia diferenças estatisticamente significativas entre os estilos de aprendizagem dos discentes e o desempenho por eles auferidos nas avaliações em grupo, individual, CRA semestral e geral, realizou-se o teste de Wilcoxon, conforme evidenciado na Tabela 5.

\begin{tabular}{lcccc}
\hline & \multicolumn{4}{l}{ Dimensão dos estilos de aprendizagem } \\
\cline { 2 - 5 } & $\begin{array}{l}\text { Processamento } \\
\text { Ativo/Reflexivo }\end{array}$ & $\begin{array}{l}\text { Percepção } \\
\text { Sensorial/Intuitivo }\end{array}$ & $\begin{array}{l}\text { Retenção } \\
\text { Visual/Verbal }\end{array}$ & $\begin{array}{l}\text { Compreensão } \\
\text { Sequencial/Global }\end{array}$ \\
\hline Avaliação em grupo & & & & \\
\hline Wilcoxon & 1345,50 & 1096,00 & 712,00 & 1476,50 \\
\hline & & & & \\
(Continua)
\end{tabular}


(Conclusão)

\begin{tabular}{lcccc}
\hline p-valor & 0,6493 & 0,3886 & 0,9484 & 0,5800 \\
\hline Avaliação Individual & & & & \\
\hline Wilcoxon & 1149,50 & 1082,5 & 663,5 & 1023,5 \\
p-valor & 0,0971 & 0,4460 & 0,629 & 0,0240 \\
\hline CRA Semestral & & & & \\
\hline Wilcoxon & 1287,00 & 115000 & 591,00 & 1154,00 \\
p-valor & 0,4157 & 0,2072 & 0,2677 & 0,1485 \\
\hline CRA Geral & & & & \\
\hline Wilcoxon & 1496,00 & 1080,00 & 793,50 & 1159,50 \\
p-valor & 0,6428 & 0,4572 & 0,5289 & 0,1583 \\
\hline
\end{tabular}

Fonte: Elaborada a partir dos dados da pesquisa.

Os resultados encontrados por meio do teste de Wilcoxon, ao nível de significância de $5 \%$, demonstram que não há influência dos estilos de aprendizagem no desempenho auferido pelos discentes, seja nas notas obtidas na avaliação em grupo, CRA semestral e CRA geral, não sendo possível afirmar que o estilo do aluno tem alguma influência sobre o resultado de tais avaliações. Assim, pode-se inferir que as variáveis em questão (estilo e desempenho) são independentes entre si, convergindo para os achados dos estudos de Cordeiro e Silva (2012), Nogueira et al. (2012) e Pellón et al. (2013).

Vale destacar que apenas no desempenho da avaliação individual, para a dimensão compreensão, identificou-se diferença significativa, visto que os discentes com estilo sequencial obtiveram médias superiores quando comparados com os de estilo global. Tal resultado indica que o estilo sequencial tem relação com as avaliações do tipo somativas (individuais - ao final dos conteúdos). Os discentes com estilo sequencial necessitam de uma sequência lógica de etapas e têm característica de pensamento convergente, ou seja, buscam uma única alternativa para resolução dos problemas e fazem isso "passo a passo". Assim, ter auferido desempenho superior nas atividades individuais demonstra a facilidade que o discente tem em seguir uma lógica quando esta desenvolve atividades individual.

Importante ressaltar que, na análise descritiva, os dados revelaram a predominância do estilo ativo, ou seja, de estudantes que se adaptam bem a estudos em grupo e dinâmicas, mas não ocorreram diferenças estatísticas significativas quando comparada à dimensão processamento para avaliações individuais e em grupo. $\mathrm{Na}$ dimensão compreensão, em que ocorreu diferença significativa na avaliação individual, os resultados descritivos indicaram que o estilo sequencial apresentou predominância, indicando que os alunos assimilam informações fragmentadas e superficiais, buscam informações e tomam decisões lineares (Felder \& Silverman, 1988).

\section{CONSIDERAÇÕES FINAIS}

0 presente estudo teve como objetivo principal verificar se 0 estilo de aprendizagem impacta no rendimento acadêmico nas avaliações dos alunos que cursaram a disciplina Análise de Custos no curso de graduação em Ciências Contábeis no que tange às avaliações formativas e somativas. Para atender o objetivo da pesquisa, identificaram-se os estilos de aprendizagem dos discentes por meio do ILS de Felder e Soloman (1991). Quanto às medidas de rendimento acadêmico, utilizou-se a nota da disciplina, bem como o CRA semestral e geral dos discentes.

Assim, constatou-se a predominância, entre os discentes pesquisados, do estilo ativo, seguido pelo sensorial, visual e sequencial, o que corrobora com os estudos anteriores da área contábil. Em relação à análise de cada estilo e rendimento acadêmico, verificou-se que tanto a nota da disciplina quanto o CRA geral e semestral apresentaram médias próximas, confirmando pelo teste estatístico que, na maioria dos casos, não há diferenças entre as variáveis analisadas.

Destaca-se que houve diferença significativa apenas entre a nota individual e 0 estilo sequencial. Entende-se que tal relação na avaliação somativa possa se dar pelo fato de que, nessa modalidade, as atividades são realizadas individualmente. Na dimensão compreensão, o estilo sequencial apresentou predominância, podendo-se inferir que os alunos assimilam informações fragmentadas e superficiais, buscam informações e tomam decisões lineares, podendo o estilo de aprendizagem afetar os resultados obtidos nas avaliações. 
Deve-se mencionar também que, na disciplina analisada, houve uma diversificação das tarefas avaliativas, provas, estudo de caso, exercícios, debates, trabalhos de vídeo e reportagem, abrangendo, de alguma forma, os diferentes estilos de aprendizagem dos alunos. Entende-se que essa diversificação pode ter contribuído para que os estilos dos alunos não impactassem no seu rendimento acadêmico, uma vez que tais atividades favoreceram o desenvolvimento de habilidades próprias de cada estilo de aprendizagem.

Assim, ressalta-se que, embora os resultados deste estudo, de modo geral, não tenham constatado impacto dos estilos de aprendizagem no rendimento acadêmico auferido, é relevante conhecê-los no processo de ensino, seja para adequação dos métodos de ensino utilizados pelo docente ou para o planejamento do próprio discente para melhorias de sua aprendizagem. Cordeiro e Silva (2012) reforçam a importância de os professores conhecerem as preferências (estilos) individuais dos estudantes e propor não só atividades que reforcem o que foi ensinado, mas incentivar o desenvolvimento de novas estratégias de aprendizagem.

Ao considerar os diferentes estilos de aprendizagem presentes em uma sala de aula, o professor terá condições de planejar e desenvolver suas atividades, contemplando a diversidade de características dos alunos, o que poderá contribuir para um melhor processo de ensino-aprendizagem, podendo contribuir também para 0 maior envolvimento dos alunos nas atividades e fazendo com que esses se sintam mais reconhecidos no ambiente de ensino e, assim, participem mais do próprio aprendizado.

Importa destacar a limitação deste estudo de se utilizar uma amostra não probabilística exclusivamente com alunos de uma disciplina oferecida no curso de graduação em Ciências Contábeis, o que não permite a generalização dos resultados. Todavia, esse aspecto não restringe a relevância dos achados evidenciados na pesquisa e poderá servir de comparação com outros estudos. Para futuras pesquisas, sugere-se a replicação do estudo em outras disciplinas do curso, bem como em outras áreas do conhecimento para comparação dos resultados.

\section{REFERÊNCIAS}

Albuquerque, L. S., Nunes, H. F. R. A., Batista, F. F., Luz, J. R. M., \& Carvalho, J. R. M. (2015) Análise dos estilos de aprendizagem dos discentes do curso de Ciências Contábeis da UFCG a partir do Inventário de Kolb. Anais do Congresso USP de Iniciação Científica em Contabilidade, São Paulo, SP, Brasil, 12.

Borges, L. F. M. (2016). Estilos e estratégias de aprendizagem: um estudo com discentes do curso de Ciências Contábeis. Dissertação de mestrado, Universidade Federal de Uberlândia, Uberlândia, MG, Brasil.

Borges, I. M. T., Santos, A., Abbas, K., Marques, K. C. M., \& da Fonseca Tonin, J. M. (2014). Reprovação expressiva na disciplina de contabilidade de custos: quais os possíveis motivos?. Revista de Educação e Pesquisa em Contabilidade (REPeC), 8(4).

Cavalcanti Neto, A. L. G. \& Aquino, J. L. F. (2009). A avaliação da aprendizagem como um ato amoroso: o que 0 professor pratica? Educação em Revista,25 (2), 223-240.

Cerqueira, T. C. S. (2000). Estilos de aprendizagem em Universitários. Tese de doutorado, Faculdade de Educação da Universidade Estadual de Campinas, São Paulo, SP, Brasil.

Corbucci, P. R. (2007) Desafios da educação superior e desenvolvimento no Brasil. IPEA, Instituto de Pesquisa Econômica Aplicada, texto para discussão n.1287. Recuperado em 15 de março, 2017, de http://www.ipea.gov.br/portal/index.php?option=com_content\&view=article\&id=4846

Cordeiro, R. A., \& da Silva, A. B. (2012). Os estilos de aprendizagem influenciam o desempenho acadêmico dos estudantes de finanças? Revista de Administração da UFSM, 5(2), 243-261.

Dias, G. P. P., Sauaia, A. C. A. \& Yoshizaki, H. T. Y. (2013) Estilos de aprendizagem Felder-Silverman e 0 aprendizado com jogos de empresa. Revista de Administração de Empresas, 53(5), 469-484, set/out.

Faria, P. M. O., \& Leal, E. A. (2014). Análise da adoção de conteúdos relacionados à área gerencial nos cursos de ciências contábeis: um estudo no estado de Minas Gerais. Anais do Congresso UFSC de Controladoria e Finanças e Iniciação em Contabilidade, Santa Catarina, SC, Brasil, 5.

Fávero, L. P., Belfiore, P., Silva, F. L. \& Chan, B. L. (2009) Análise de dados: modelagem multivariada para tomada de decisões. Rio de Janeiro: Elsevier. 
Felder, R. M., \& Silverman, L. K. (1988). Learning and teaching styles in engineering education. Journal of Engineering Education, 78(7), 674-681.Recuperado em 7 de julho, 2015 de http://www.ncsu.edu/felderpublic/Learning_Styles.html

Felder, R. M., \& Soloman, B. A. (1991). Index of Learning Styles Questionnaire .North Carolina State University. Recuperado em 7 de julho, 2015 de https://www.engr.ncsu.edu/learningstyles/ilsweb.html

Ferreira, M. C., Assmar, E. M. L., Omar, A. G., Delgado, H. H., González, A. T., Souza, M. A., \& Cisne, M. C. F. (2002). Atribuição de causalidade ao sucesso e fracasso escolar: um estudo transcultural Brasil-ArgentinaMéxico. Psicologia: Reflexão e Crítica, 15(3), 515-527.

Garcia, J. (2009). Avaliação e aprendizagem na educação superior. Estudos em Avaliação Educacional, 20 (43), 201-213.

Gonçalves, L. F. (2005). Programa de avaliação do sistema educacional do estado do Paraná-AVA-1995/2002: uma avaliação a serviço da formação humana, ou de favorecimento ao mercado econômico? Dissertação de mestrado, Universidade Federal do Paraná, Curitiba, PR, Brasil.

Lopes, W. M. G. (2002) Inventário de Estilos de Aprendizagem de Felder-Saloman: investigação de sua validade em estudantes universitários de Belo Horizonte. Dissertação de Mestrado, Universidade Federal de Santa Catarina, SC, Brasil.

Meurer, A. M.; Pedersini, D. R.; Antonelli, R. A. Voese, S. B. Estilos de Aprendizagem e Rendimento Acadêmico: uma análise dos acadêmicos e professores de Ciências Contábeis. Anais do Congresso UFSC de Controladoria e Finanças, Florianopólis, SC, Brasil, 7.

Miranda, G. J., Lemos, K. C. S., Oliveira, A. S., \& Ferreira, M. A. (2015) Determinantes do desempenho acadêmico na área de negócios. Meta: Avaliação, 7(20), p.175-209.

Munhoz, A. M. H. (2004). Uma análise multidimensional da relação entre inteligência e desempenho acadêmico em universitários ingressantes. Tese de doutorado, Universidade Estadual de Campinas, Faculdade de Educação, Campinas, SP, Brasil.

Nogueira, D. R., Espejo, M. M. S. B., Reis, L. G., \& Voese, S. B. (2012). Estilos de aprendizagem e desempenho em educação a distância: um estudo empírico com alunos da $s$ disciplinas de Contabilidade Geral e Gerencial. Revista de Educação e Pesquisa em Contabilidade, 6(1), 54-72.

Pellón, M., Nome, S., \& Arán, A. (2013). Relação entre estilos de aprendizagem e rendimento acadêmico dos estudantes do quinto ano de medicina. Revista Brasileira de Oftalmologia, 72(3), 181-184.

Santos, J. F. S. (2005) Avaliação no ensino a distância. Revista Ibero-americana de Educação, 38 (4), 1-9.

Santos, N. A. (2012). Determinantes do desempenho acadêmico dos cursos de ciências contábeis. Tese de doutorado. Programa de Pós-Graduação em Ciências Contábeis, Departamento de Contabilidade e Atuária, FEA/USP, São Paulo, SP, Brasil.

Shaftel, J., \& Shaftel, T. L. (2007). Educational Assessment and the AACSB. Issues in Accounting Education, 22(2), 215-232.

Silva, D. M. (2006). O impacto dos estilos de aprendizagem no ensino de Contabilidade na FEA-RP/USP. Dissertação de mestrado, Universidade de São Paulo, Ribeirão Preto, SP, Brasil.

Silva, D. M., Leal, E. A., Pereira, J. M., \& Oliveira, J. D. Neto (2015). Estilos de aprendizagem e desempenho acadêmico na Educação a Distância: uma investigação em cursos de especialização. Revista Brasileira de Gestão de Negócios, 17(57), 1300-1316.

Silva, D. M., \& Oliveira, J. D. Neto (2010). O impacto dos estilos de aprendizagem no ensino de Contabilidade. Contabilidade Vista \& Revista, 21(4), 123-156.

Silva, L. L. V. (2012). Estilos e estratégias de aprendizagem de estudantes universitários. Dissertação Mestrado, Universidade de São Paulo, São Paulo, SP, Brasil.

Souza, E. S. (2008). ENADE 2006: Determinantes do Desempenho dos cursos de Ciências Contábeis. Dissertação. Mestrado. Programa Multiinstitucional e Inter-Regional de Pós- Graduação em Ciências Contábeis, Brasília, DF, Brasil. 
Souza, L. M. D., Avelino, B. C., \& Takamatsu, R. T. (2017). Estilos de aprendizagem e influência no processo de ensino-aprendizagem: Análise empírica na visão de estudantes de contabilidade. Revista Ambiente Contábil, 9(2), 379-400

Stake, R. E. (1967). The countenance of educational evaluation. Department for Exceptional Children, Gifted Children Section.

Tyler, R. W. (1942). General Statement on Evaluation, The Journal of Educational Research, 35(7), 492-501.

Vianna, H. M. (1982). Avaliação educacional-problemas gerais e formação do avaliador. Educação e Seleção, 5. $9-14$. 